P444 CHANGING PRACTICES IN A LARGE URBAN HIV CLINIC IN RESPONSE TO COVID-19

A Keane* , F Lyons, A Loy. GUIDe Department St James Hospital, Dublin, Ireland

10.1136/sextrans-2021-sti.460

Background/Purpose In March 2020 the World Health Organisation declared Covid-19 a global pandemic. Ireland responded by shutting all non-essential shops, businesses and schools. Our healthcare system had to adapt and rapid adaptations were made to ambulatory HIV care.

Approach Records were virtually reviewed and triaged using existing electronic patient records(EPR) prior to appointments. Antiretrovirals(ARVs) were pre-prescribed and prepared by pharmacy for those anticipated to remain on the same ARVs. We determined those for whom routine phlebotomy could be diverted. We paused cervical, STI screening and vaccination programme in the initial phase, reopening in a more streamlined manner as the pandemic went out of each wave and adapting again as it went back into a new wave. We undertook a staff survey to assess their views on the new service delivery.

Outcomes/Impact We conducted a survey of our staff members across all disciplines. There were 34 responses out of 53 staff members. $76.47 \%$ agreed that pre reviewing EPR was better for patient care, $88.24 \%$ believed that patient flow was improved, $76.47 \%$ believed that patients time in the department was reduced due to the virtual reviews and that covid19 risk was reduced. $80 \%$ of doctors felt that doing the virtual reviews was a valuable training experience. 50\% believed that there was more time available to deal with complex cases.

Innovation and Significance Pre prescribing medications has led to better patient flow and social distancing during clinic. Complex issues, in many cases can be addressed prior to clinic and discussed with senior providers. Stricter adherence to appointment times is more convenient for patients and staff. This system has allowed us to identify patients with complex medical needs and presented opportunity for education and training between senior and junior providers, whilst decreasing Covid-19 risk to provider and patient.

\section{P445 FORMALIZING PARTNERSHIPS: HOW REGIONAL COALITIONS IN SEXUAL HEALTH CAN ADDRESS THE STI EPIDEMIC}

A Riba*, A Landa, T Anderson, L Potyondy, E Starzyk, K Wendel. Denver Prevention Training Center, Denver, USA

10.1136/sextrans-2021-sti.461

Background Sexually transmitted infection (STI) rates have increased in the United States and in Colorado for over five years. To address the rising STI rates in the Denver Metro Area (DMA), leaders at the Denver Prevention Training Center (DPTC), Denver Public Health, and the Colorado Department of Health and Environment (CDPHE) supported the establishment of the Denver Metro STI Coalition (DMSC), a collective impact group. Collective impact groups have a history of success in aiding public health crises, most notably the HIV epidemic, but have not been extensively used to address the ongoing U.S. STI epidemic. We discuss the development of an STI collective impact group to increase local communication and collaboration to support high quality, accessible STI care and reduce STI health disparities in our jurisdiction.

Approach DMSC members were recruited from health care and community-based organizations engaged in STI services in DMA. This report summarizes DMSC progress from its initial meeting on $11 / 2019$ to $3 / 2021$.

Outcomes/Impact DMSC participation has included 106 individuals from 20 health care and community-based organizations. DMSC has conducted four quarterly general assembly meetings. DMSC has facilitated member education through its quarterly meetings, group emails, and DMSC Groupsite shared documents. Shared education and resources have included information on STI epidemiology, STI testing shortages, CDC STI updates, and local STI health alerts. DMSC workgroups have developed products including a one-page congenital syphilis infographic highlighting jurisdictional epidemiology and treatment algorithms, including state specific recommendations for universal third trimester syphilis screening during pregnancy and a provider counseling guide for safe sex during COVID-19.

Innovation The DMSC has provided a structure for leveraging local partnerships, sharing information, expanding healthcare provider STI knowledge and improving STI infrastructure in the metropolitan area. STI focused collective impact groups could be used in other jurisdictions to coordinate efforts to control rising STI rates.

\section{P446 DECREASED SEX PARTNERSHIPS BUT NO CHANGE IN GONORRHEA/CHLAMYDIA PREVALENCE AMONG GAY, BISEXUAL AND OTHER MEN-WHO-HAVE-SEX-WITH-MEN (MSM) DURING THE COVID-19 PANDEMIC}

C Schumacher*, N Thornton, C Tilchin, K Ghanem, M Hamill, A Rompalo, C Latkin, S Ruhs, S Rives, J Jennings. Johns Hopkins University School of Medicine, Baltimore, USA

10.1136/sextrans-2021-sti.462

Background COVID-19 mitigation measures may indirectly impact sexually transmitted infection (STI) transmission. Social distancing may impact number/type of sex partnerships and access to STI testing and treatment. The objective was to compare the number of reported sex partnerships and gonorrhea and chlamydia prevalence pre- and during-pandemic among a cohort of gay, bisexual and other men-who-have-sexwith-men (MSM) in Baltimore, Maryland, a U.S. city with sustained STI epidemics.

Methods This study was nested in a cohort study of sexually active MSM aged 18-45, and included participants who had at least one study visit after March 13, 2020 (during-pandemic) and $\leq$ six months between their first during-pandemic and last pre-pandemic visit. Wilcoxon and McNemar tests for paired data were used for statistical testing.

Results Among 417 MSM enrolled in the cohort, 220 (52.8\%) were included. 213 (96.8\%) had a visit between April 6-June 30, 2020 (during-pandemic1); 185 (84.1\%) had a visit between July 1-September 30, 2020 (during-pandemic2), including seven who missed the during-pandemic1 visit. The majority were Black (73.2\%) and aged 24-35 (56.4\%); 42\% were living with HIV. Compared to pre-pandemic, the median number of total and casual male sex partners (past three months) significantly declined during-pandemic1 and duringpandemic2 (Total partners: pre-pandemic: 2.0 (range: 0-75); during-pandemic1: 1.0 (0-25), $\mathrm{p}<0.0001$; during-pandemic2: 